\title{
The Reflection of Principal Leadership on Teacher Outcomes: An Analysis of Turkish and Foreign Studies
}

\author{
İzlem Gözükara ${ }^{1}$ \\ ${ }^{1}$ Department of Business Administration, Istanbul Arel University, Turkey \\ Correspondence: Izlem Gozukara, Istanbul Arel University, Turkey.
}

Received: January 28, 2021

Accepted: April 22, $2021 \quad$ Online Published: May 12, 2021

doi:10.5539/ibr.v14n6p33

URL: https://doi.org/10.5539/ibr.v14n6p33

\begin{abstract}
Leadership continues to be one of the most discussed topics in the field of management and there are ongoing studies regarding its types and effectiveness in several kinds of organizations. Among others, educational institutions have gained considerable interest from researchers due to the significance of education in a society. In educational context, elementary education has a critical place in terms of guiding all academic education. From this point of view, this paper seeks to determine which types of leadership related to elementary schools have been commonly investigated in the last six years both in Turkey and at abroad, and which outcomes related to teachers have been associated with those leadership styles. Therefore, the purpose of this paper is to offer an insight into the recent trend in educational management literature and provide a brief summary of recent studies in this regard, which may be useful for future researchers.
\end{abstract}

Keywords: principal leadership, teacher outcomes, elementary education, school management

\section{Introduction}

Development of managerial systems determines the degree of social developments. Managers and employees, therefore, are of capital importance. Management represents the way of coordinating both material and human resources in order to accomplish the goals of an organization in a manner that is socially acceptable (Jasbi, 2011). Leadership constitutes one of the crucial management tasks and it incorporates the art and science of affecting other people for the sake of goal achievement (Khalkhali et al, 2011).

Success is primarily based on the manager or the supervisor of any organization such as business enterprises or educational institutions. Thus, improved success is highly affected by leaders and their leadership styles. Leadership is a phenomenon utilized by organizations to modify attitudes and behaviors of their employees. It emphasizes the power to influence others and make them act in line with certain purposes and targets (Şişman, 2012). As a result, the most effective type of leadership among many leadership styles and approaches cannot be definitely pointed out as it may vary by several factors such as traits, conditions, and the type and goals of the organization or culture.

Leadership can be defined as the process of influencing the behaviors of a group of people in order to achieve a target (Nworgu, 1991). In educational context, it refers to ensure that the business is carried out with the support and cooperation of other people within the school system (Aghenta, 2001). Schools are places where learning is realized, education service is produced and provided, which brings the challenge to adapt to the environmental conditions that are changing and evolving on a continuous basis. To achieve this purpose, principals and teachers have to undertake serious responsibilities in all levels of the education system and adopt certain leadership behaviors. Nevertheless, school principals, due to their position, have a key leadership position. Thus, the leadership styles and behaviors of school principals have prime importance for both the entire school system and the society. Such leadership behaviors have an impact on all elements of the school, especially on teachers.

The aim of the present paper is to explore the studies conducted on principal leadership and its consequences related to teachers. For this purpose, this paper chose 10 studies each from Turkey and abroad, which discussed principal leadership and various teacher outcomes, covering the period from 2010 to 2016. 


\section{Principal Leadership and Teacher Outcomes}

Schools are organizations for learning that contribute to information development of a society. Fulfillment of individual and social requirements starts with the elementary level of educational life. Elementary education provides both a basic and a mandatory education service as well as represents the onset of academic learning.

The socioeconomic and technological progress is continuous and intensive; thereby it has created the need to reconfigure educational practices, too. The challenges due to such need do not only involve the improvement of educational outcomes, but also the adaptation to the new requirements of the society. Accordingly, schools have to implement necessary changes in order to increase the quality of education and enhance the learning capacity both at school and student levels. At this point, as the leaders of the school, principals incur the greatest responsibility because they are school administrators in charge of structuring schools in line with social, technological, and scientific requirements, and resolving potential issues (Başaran, 2000). Since principals have influence over all components of the schools, they also have a critical role in enhancing school performance and realizing changes (Leithwood \& Steinbach, 1993; Şişman, 2002).

A school administrator's level of competency in corporate leadership, and knowledge and capacity in creating an educational environment are among the main factors for achieving organizational goals of a school. The resolution of issues of educational institutions mostly depends on the extent to which a school principal performs leadership roles and behaviors (Çelik, 1999).

The principal is considered as the leader of teachers, students and other staff of a school. Leadership has a close relationship with communication and experience with people in an organized group. Principals manifest their style of leadership in their behaviors through which they are accepted as the leader of their group. The common opinion regarding schools is that the an effective leadership of administrators is the most contributive factor for school success even the school is well-equipped with a solid educational program, competent teachers and many facilities, because a leader is needed to manage and coordinate all components and progress. The style of principal identifies the culture, environment, policies, and strategies of the school. Principals' leadership styles consist of certain traits, attitudes and skill sets basically resulting from values, trust, leadership orientation and the feeling of security in significant circumstances (Ming-Ten et al., 2011). Principals' interaction with their staff is manifested through their style of leadership (Pirhaefi, 2009). Therefore, every principal's behaviors are exhibited in a certain manner that differs from others. Such consistent behavioral pattern is referred to as the leadership style of a principal.

School leadership requires guidance, support, and behaviors necessary for changing the overall success of a school. Leadership is an evolving and effective process that enables to achieve the desired outcome (Yukl, 2002). It involves affecting and supporting others for realizing a vision built upon certain personal and professional values. School leadership practices may alter the academic path of a school. According to Danielson (2010), school transformation is connected with the collaboration of principals, teachers, school, and parents.

A good principal leads to both a school with success and teachers with efficient performance (Ilgar, 2000). The performance of teachers refers to the assignments they carry out during a particular period within the school system in order to accomplish organizational goals (Obilade, 1991). It can also be defined as teachers' capacity to integrate relevant inputs to improve education-training processes (Okeniyi, 1995) or the extent to which teachers participate in daily operation of the school (Peretemode, 1991). As different conditions may drive individuals to act differently, teachers are likely to exhibit a more efficient performance when school principals establish their needs and try to meet them.

Teachers are the core component of academic success on which school performance centers. School leadership ensures the management and flow of cultural information that will support students' academic development and teachers' professional development. In this context, action-oriented and response-centered school leaders have been shown to be effective in turning teachers into role models who brings value and success in teaching (Farr, 2011; Townsend, 2010).

Irrespective of the model or approach, school leadership primarily aims to achieve and maintain school improvement. Such improvement is related to the quality of education, which is the factor with the greatest effect on students' success. Greater performance is one of the most important goals of leaders, which they make various efforts to achieve considering individuals' beliefs, values, skills, and motivations (Leithwood, 2006). School leaders are capable of motivating teachers toward improved performance by means of establishing a clear vision. A common vision that is clearly defined in terms of roles, targets, and expectations is likely to enhance the in-class efficiency of teachers. 
The review of educational management reveals that several types of leadership have been discussed. This paper, rather than focusing on a single type, explored which leadership types have been examined and associated with teacher outcomes over a six-year period. The following section will present the studies identified from literature review and discuss their results in detail.

\section{Studies on Principal Leadership and Teacher Outcomes}

The leadership behaviors of principals and the associated teacher outcomes have been examined by several studies over the last decade all around the world. In Turkey, the topics studied regarding leadership mostly include ethical leadership (e.g. Madenoğlu et al., 2014), transformational leadership (e.g. Töremen \& Yasan, 2010), cultural leadership (e.g., Balkar, 2015), instructional leadership (e.g. Taşdelen et al., 2015) and paternalistic leadership (e.g. Cerit, 2012a). The variables studied regarding teachers, in turn, include perceptions of organizational justice (e.g. Karaman \& Çankaya, 2015), motivation and job satisfaction (e.g. İnandı et al., 2010) and professional performance (e.g. Çimen, 2015). For the purpose of this paper, 10 articles conducted in Turkey were chosen and presented in Table 1. Articles are shown in a chronological order and necessary details on the research are provided.

Table 1. Turkish Studies on Principal Leadership and Teacher Outcomes

\begin{tabular}{|c|c|c|c|c|c|}
\hline Purpose & $\begin{array}{l}\text { Research } \\
\text { Design }\end{array}$ & Sample & $\begin{array}{l}\text { Data } \\
\text { Collection }\end{array}$ & Data Analysis & Findings \\
\hline \multicolumn{6}{|c|}{$\begin{array}{l}\text { Sağır, M., \& Memişoğlu, S.P. (2012). The perceptıons of administrators and teachers on elementary school administrators' educational } \\
\text { leadership roles. Journal of Research in Education and Teaching, 1(2), 1-12. }\end{array}$} \\
\hline $\begin{array}{l}\text { To reveal the administrator } \\
\text { and teacher perceptions } \\
\text { Instructional Leadership } \\
\text { Behaviors Scale about } \\
\text { instructional leadership roles } \\
\text { of the elementary school } \\
\text { administrators. }\end{array}$ & $\begin{array}{l}\text { Descriptive } \\
\text { survey }\end{array}$ & $\begin{array}{l}1783 \text { elementary } \\
\text { school teachers } \\
\text { and } 102 \\
\text { elementary } \\
\text { school } \\
\text { administrators } \\
\text { from } 45 \text { schools } \\
\text { in } 15 \text { districts of } \\
\text { Istanbul }\end{array}$ & $\begin{array}{l}\text {-Instructional } \\
\text { Leadership } \\
\text { Behaviors Scale } \\
\text { for School }\end{array}$ & T-test & $\begin{array}{l}\text { There was a significant } \\
\text { difference in perceptions of } \\
\text { administrators and teachers } \\
\text { about instructional leadership } \\
\text { behaviors of elementary school } \\
\text { administrators. }\end{array}$ \\
\hline \multicolumn{6}{|c|}{$\begin{array}{l}\text { Cerit, Y. (2012b). Lider üye etkileşimi ile öğretmenlerin performansları arasındaki ilişki (The Relationship between leader-member exchange } \\
\text { and teachers' performance). Balikesir University The Journal of Social Sciences Institute, 15(28), 33-45. }\end{array}$} \\
\hline $\begin{array}{l}\text { To investigate the } \\
\text { relationship between } \\
\text { leader-member exchange and } \\
\text { teachers' performance. }\end{array}$ & $\begin{array}{l}\text { Relational } \\
\text { survey }\end{array}$ & $\begin{array}{l}253 \text { elementary } \\
\text { school teachers in } \\
\text { Bolu }\end{array}$ & $\begin{array}{l}\text {-Effective } \\
\text { Leader-Member } \\
\text { Exchange Scale } \\
\text {-Performance } \\
\text { Scale }\end{array}$ & $\begin{array}{l}\text {-Correlation } \\
\text {-Regression }\end{array}$ & $\begin{array}{l}\text { There was a significant and } \\
\text { positive correlation between } \\
\text { leader-member exchange and } \\
\text { teachers' performance. } \\
\text { Leader-ember exchange was } \\
\text { shown to significantly explain } \\
\text { teachers' performance. }\end{array}$ \\
\hline
\end{tabular}

Teyfur, M., Beytekin, O.F., \& Yalçınkaya, M. (2013). A research on the ethical leadership of primary school administrators and the organizational trust levels in primary schools: The sample of İzmir. Dicle Üniversitesi Ziya Gökalp Ĕ̈itim Fakültesi Dergisi, $21,84-106$.

\begin{tabular}{|c|c|c|c|c|c|}
\hline $\begin{array}{l}\text { To examine the effect of } \\
\text { ethical leadership of } \\
\text { elementary } \\
\text { administrators } \\
\text { school } \\
\text { organizational trust } \\
\text { schools. }\end{array}$ & $\begin{array}{l}\text { Relational } \\
\text { survey }\end{array}$ & $\begin{array}{l}716 \text { elementary } \\
\text { school teachers in } \\
\text { İzmir }\end{array}$ & $\begin{array}{l}\text {-Ethical } \\
\text { Leadership } \\
\text { Scale } \\
\text {-Organizational } \\
\text { Trust in School } \\
\text { Scale }\end{array}$ & $\begin{array}{l}\text {-Regression } \\
\text {-Mann-Whitney } \\
\text { U test } \\
\text {-t-test for } \\
\text { independent } \\
\text { groups } \\
\text {-Kruskal Wallis } \\
\text { test }\end{array}$ & $\begin{array}{l}\text { Teachers reported that the } \\
\text { ethical leadership skills of their } \\
\text { school administrators } \\
\text { significantly affect their } \\
\text { organizational trust levels. }\end{array}$ \\
\hline
\end{tabular}

Aybek, B., Titiz, H. ve Gümüşay, T. (2014). İlkokul müdürlerinin etkili liderlik düzeylerine ilişkin öğretmen görüşlerinin incelenmesi (Examination of teacher opinions about the effective leadership levels of elementary school principals). Journal of Research in Education and Teaching, 3(4). 342-355.

\begin{tabular}{|c|c|c|c|c|c|}
\hline $\begin{array}{l}\text { To establish teachers' } \\
\text { opinions about the levels of } \\
\text { effective leadership } \\
\text { competency of elementary } \\
\text { school principals and } \\
\text { determine whether such } \\
\text { opinions are different in } \\
\text { terms of several variables } \\
\text { (age, gender, educational } \\
\text { status, seniority) }\end{array}$ & $\begin{array}{l}\text { Descriptive } \\
\text { survey }\end{array}$ & $\begin{array}{lr}452 & \text { teachers } \\
\text { from } 18 \text { schools } \\
\text { in Adana }\end{array}$ & $\begin{array}{l}\text { Effective } \\
\text { Leader Quality } \\
\text { Scale }\end{array}$ & $\begin{array}{l}\text {-Frequency } \\
\text { analysis } \\
\text { - t-test } \\
\text {-One-Way } \\
\text { ANOVA }\end{array}$ & $\begin{array}{l}\text { Teachers reported that principals } \\
\text { "always" exhibited effective } \\
\text { leader behaviors. } \\
\text { There was no significant } \\
\text { difference in teachers' opinions } \\
\text { in terms of age, gender, } \\
\text { educational status, seniority, and } \\
\text { total time of duty. }\end{array}$ \\
\hline
\end{tabular}


Ayık, A., \& Şayir, G. (2014). İlköğretim kurumlarında görevli okul müdürlerinin öğretimsel liderlik davranışlarının çeşitli değişkenler açısından incelenmesi (Examination of instructional leadership behaviors of principals working at elementary schools in terms of several variables). Ekev Akademi Dergisi, 18(60), 15-30.

\begin{tabular}{|c|c|c|c|c|c|}
\hline $\begin{array}{l}\text { To determine the extent of } \\
\text { instructional leadership } \\
\text { behaviors of elementary } \\
\text { school principals, and } \\
\text { whether teachers' opinions } \\
\text { about instructional } \\
\text { leadership vary by gender, } \\
\text { seniority and field of study. }\end{array}$ & $\begin{array}{l}\text { Descriptive, } \\
\text { Relational } \\
\text { survey }\end{array}$ & $\begin{array}{l}290 \text { elementary } \\
\text { school teachers in } \\
\text { Konya, Meram }\end{array}$ & $\begin{array}{l}\text { "Instructional } \\
\text { Leadership } \\
\text { Behaviors of } \\
\text { Principals" } \\
\text { Questionnaire }\end{array}$ & $\begin{array}{l}\text {-t-test } \\
\text {-ANOVA } \\
\text {-Kruskal Wallis } \\
\text { test }\end{array}$ & $\begin{array}{l}\text { The dimensions perceived in the } \\
\text { highest level were teaching } \\
\text { process and evaluation of the } \\
\text { students. } \\
\text { The dimensions perceived in the } \\
\text { lowest level were supporting } \\
\text { and improving teachers. } \\
\text { Teachers' perceptions about } \\
\text { exhibiting IL behaviors did not } \\
\text { significantly vary by gender; but } \\
\text { social and fine arts teachers had } \\
\text { greater perceptions compared to } \\
\text { others. }\end{array}$ \\
\hline
\end{tabular}

Korkmaz, İ. (2015). Öğretmenlerin mesleki gelişimlerinde okul yöneticileri ve denetmenlerin etkililiğinin incelenmesi (Examination of the efficacy of school administrators and supervisors in teachers' professional development). Uluslararası Eğitim Bilimleri Dergisi, 2(4), 55-64.

\begin{tabular}{l|l|l|l|l|l}
$\begin{array}{l}\text { To examine the efficacy of } \\
\text { school administrators and } \\
\text { supervisors in teachers' } \\
\text { interactions providing } \\
\text { professional development. }\end{array}$ & Qualitative & 94 class teachers & $\begin{array}{l}\text { Open ended } \\
\text { questions }\end{array}$ & $\begin{array}{l}\text { Content } \\
\text { analysis }\end{array}$ & $\begin{array}{l}\text { School administrators generally } \\
\text { focus on the fulfillment of } \\
\text { official responsibilities by } \\
\text { teachers; however teachers } \\
\text { expect the recognition of their } \\
\text { in-class activities, performance, } \\
\text { and efforts to increase their } \\
\text { students' success. }\end{array}$ \\
\hline
\end{tabular}

Yeșilyurt, R. (2015). Okul müdürlerinin etkileşimci liderlik stilleri ile öğretmenlerin okula bağlllı düzeyleri arasındaki ilişsi (The relationship between transactional leadership styles of principals and teachers' commitment to school). Yüksek Lisans Tezi (Master's Thesis). Eskișehir Osmangazi Üniversitesi, Eğitim Bilimleri Enstitüsü, Eskișehir.

\begin{tabular}{|c|c|c|c|c|c|}
\hline $\begin{array}{l}\text { To investigate the } \\
\text { relationship between } \\
\text { principals' transactional } \\
\text { leadership style and } \\
\text { teachers' commitment to the } \\
\text { school based on teachers' } \\
\text { opinions. }\end{array}$ & Relational & $\begin{array}{l}506 \text { teachers } \\
\text { from elementary, } \\
\text { secondary and } \\
\text { high schools in } \\
\text { Afyonkarahisar } \\
\text { city center }\end{array}$ & $\begin{array}{l}\text {-Multifactor } \\
\text { Leadership } \\
\text { Questionnaire } \\
\text {-Organizational } \\
\text { Commitment } \\
\text { Scale }\end{array}$ & $\begin{array}{l}\text {-Frequency } \\
\text { analysis } \\
\text {-Pearson's } \\
\text { correlation } \\
\text {-One-Way } \\
\text { ANOVA } \\
\text {-Regression }\end{array}$ & $\begin{array}{l}\text { Principals exhibited } \\
\text { transactional leadership to a } \\
\text { moderate extent. } \\
\text { Teachers' school commitment } \\
\text { was the highest in affective } \\
\text { commitment dimension. } \\
\text { There was a significantly } \\
\text { positive and weak relationship } \\
\text { between principals' } \\
\text { transactional leadership and } \\
\text { teachers' school commitment } \\
\text { Teachers' school commitment } \\
\text { varied by gender, age, and }\end{array}$ \\
\hline
\end{tabular}

Yavaş Taşdelen, T., Aküzüm, C., Tan, Ç. ve Uçar, M. B. (2015). Öğretmen görüşleri açısından okul müdürlerinin öğretimsel liderlik davranışları (Instructional leadership behaviors of principals in terms of teacher opinions). International Periodical For The Languages, Literature and History of Turkish or Turkic Volume 10/3, 1-26.

\begin{tabular}{l|l|l|l|l|l|}
$\begin{array}{l}\text { To explore how instructional } \\
\text { leadership behaviors of } \\
\text { principals are shaped based } \\
\text { on teachers' opinions. }\end{array}$ & Qualitative & $\begin{array}{l}52 \text { teachers from } \\
\text { elementary, } \\
\text { secondary and } \\
\text { high schools in } \\
\text { Gaziantep Nizip }\end{array}$ & $\begin{array}{l}\text { Semi-structured } \\
\text { interview }\end{array}$ & $\begin{array}{l}\text {-NVivo } \\
\text { qualitative data } \\
\text { analysis } \\
\text { program }\end{array}$ & $\begin{array}{l}\text { Teachers perceived the } \\
\text { elementary, secondary and high } \\
\text { school principals as instructional } \\
\text { and leading The most } \\
\text { prominent positive behaviors } \\
\text { were "leading, guiding, sharing } \\
\text { experience and student-teacher } \\
\text { centeredness". }\end{array}$ \\
\hline
\end{tabular}

Ereş, F., \& Akyürek, M.İ. (2016). Relationship levels between distributed leadership behaviors of principals and the teachers' perception of job satisfaction. GEFAD/GUJGEF, 36(3), 427-449.

\begin{tabular}{l|l|l|l|l|l|}
$\begin{array}{l}\text { To establish the relationship } \\
\text { between elementary school } \\
\text { principals' distributed } \\
\text { leadership behaviors and } \\
\text { teachers' perception of job } \\
\text { satisfaction. }\end{array}$ & $\begin{array}{l}\text { Descriptive, } \\
\text { Relational } \\
\text { survey }\end{array}$ & $\begin{array}{l}296 \text { teachers in } \\
\text { Ankara }\end{array}$ & $\begin{array}{l}\text { - Distributed } \\
\text { Leadership } \\
\text { Scale } \\
\text { - Job } \\
\text { Satisfaction } \\
\text { Scale }\end{array}$ & $\begin{array}{l}\text {-t-test } \\
\text {-Analysis of } \\
\text { variance }\end{array}$ & $\begin{array}{l}\text { There was a positive } \\
\text { relationship between principals' } \\
\text { distributed leadership behaviors } \\
\text { and teachers' job satisfaction. }\end{array}$ \\
\end{tabular}

Bellibas, M.S., Bulut, O., Hallinger, P, \& Wang, W.C. (2016). Developing a validated instructional leadership profile of Turkish primary school principals. International Journal of Educational Research, 75, 115-133. 


\begin{tabular}{|c|c|c|c|c|}
\hline $\begin{array}{l}\text { To develop and validate a } \\
\text { Turkish version of the } \\
\text { Principal Instructional } \\
\text { Management Rating Scale } \\
\text { (PIMRS) Teacher Form }\end{array}$ & $\begin{array}{l}294 \text { teachers } \\
\text { from } \\
\text { elementary } \\
\text { schools in } 6 \\
\text { cities. }\end{array}$ & $\begin{array}{l}\text { Principal } \\
\text { Instructional } \\
\text { Management } \\
\text { Rating Scale } \\
\text { (PIMRS) }\end{array}$ & $\begin{array}{l}\text { Content } \\
\text { validation tests, } \\
\text { Construct } \\
\text { validation tests } \\
\text { (e.g., } \\
\text { confirmatory } \\
\text { factor analysis, } \\
\text { reliability) and } \\
\text { item-level } \\
\text { analyses (e.g., } \\
\text { Rasch } \\
\text { analysis). }\end{array}$ & $\begin{array}{l}\text { Both PIMRS Teacher Form } \\
\text { (Turkish) and PIMRS Principal } \\
\text { Form (Turkish) could be used in } \\
\text { studies of secondary school } \\
\text { principals as well as primary } \\
\text { school principals. }\end{array}$ \\
\hline
\end{tabular}

Table 2. Foreign Studies on Principal Leadership and Teacher Outcomes

\begin{tabular}{|c|c|c|c|c|c|}
\hline Purpose & Research Design & Sample & Data Collection & Data Analysis & $\begin{array}{l}\text { Findings } \\
\end{array}$ \\
\hline \multicolumn{6}{|c|}{$\begin{array}{l}\text { Finnigan, K.S. (2010). Principal leadership and teacher motivation under high-stakes accountability policies. Leadership and Policy in } \\
\text { Schools, 9(2), 161-189 }\end{array}$} \\
\hline $\begin{array}{l}\text { Investigation of } \\
\text { leadership of principals } \\
\text { and motivation of } \\
\text { teachers in schools under } \\
\text { accountability sanctions } \\
\text { based on expectancy } \\
\text { theory } \\
\text { transformational and } \\
\text { leadership. }\end{array}$ & Survey & $\begin{array}{l}\text { All } \\
\text { elementary } \\
\text { school } \\
\text { teachers in } \\
\text { the Chicago } \\
\text { Public } \\
\text { Schools } \\
\text { (USA) }\end{array}$ & $\begin{array}{l}\text {-Consortium for } \\
\text { Chicago School } \\
\text { Research's } \\
\text { (CCSR) survey } \\
\text { (one expectancy } \\
\text { construct and four } \\
\text { leadership } \\
\text { constructs } \\
\text { (instructional } \\
\text { leadership, } \\
\text { teacher-principal } \\
\text { trust, principal } \\
\text { support } \\
\text { for change, and } \\
\text { inclusive } \\
\text { leadership) }\end{array}$ & $\begin{array}{l}\text {-Percentage } \\
\text {-Correlation }\end{array}$ & $\begin{array}{l}\text {-A correlation between } \\
\text { principal instructional } \\
\text { leadership and support for } \\
\text { change and teacher } \\
\text { expectancy. } \\
\text { - A correlation between } \\
\text { teacher experience, race, } \\
\text { advanced education, } \\
\text { performance level of } \\
\text { school and teacher } \\
\text { expectancy. } \\
\text {-A correlation between } \\
\text { teacher expectancy and } \\
\text { school's capacity to leave } \\
\text { probation status. }\end{array}$ \\
\hline \multicolumn{6}{|c|}{$\begin{array}{l}\text { Adeyemi, T.O. (2010). Principals' leadership styles and teachers' job performance in senior secondary schools in Ondo State, Nigeria. } \\
\text { Current Research Journal of Economic Theory, 3(3): 84-92. }\end{array}$} \\
\hline $\begin{array}{l}\text { To examine the } \\
\text { leadership styles of } \\
\text { senior secondary school } \\
\text { principals and job } \\
\text { performance of teachers. }\end{array}$ & Descriptive & $\begin{array}{l}240 \\
\text { principals } \\
\text { and } 1800 \\
\text { teachers } \\
\text { from } 240 \\
\text { senior } \\
\text { secondary } \\
\text { schools in } \\
\text { Nigeria }\end{array}$ & $\begin{array}{l}\text {-Principals' } \\
\text { leadership style } \\
\text { questionnaire } \\
\text { (PLSQ) } \\
\text {-teachers' job } \\
\text { performance } \\
\text { questionnaire } \\
\text { (TJPQ) }\end{array}$ & $\begin{array}{l}\text { Descriptive } \\
\text { statistics } \\
\text { Correlation } \\
\text { matrix } \\
\text { t-test }\end{array}$ & $\begin{array}{l}\text { The most common } \\
\text { leadership style was } \\
\text { democratic leadership. } \\
\text { Teachers' job performance } \\
\text { was at a moderate level. } \\
\text { Teachers' job performance } \\
\text { was better in schools with } \\
\text { principals using autocratic } \\
\text { leadership style than in } \\
\text { schools with principals } \\
\text { using democratic or } \\
\text { laissez-faire leadership } \\
\text { styles. }\end{array}$ \\
\hline \multicolumn{6}{|c|}{$\begin{array}{l}\text { Graham, K., Hudson, P., \& Willis, J. (2014, December). How can principals enhance teacher job satisfaction and work commitment? Paper } \\
\text { presented at the Australian Association of Research in Education (AARE) Conference, Brisbane, Australia. }\end{array}$} \\
\hline $\begin{array}{l}\text { Examination of the effect } \\
\text { of school leadership on } \\
\text { teachers' job satisfaction } \\
\text { to reveal how principals } \\
\text { increase work } \\
\text { commitment of teachers. }\end{array}$ & $\begin{array}{l}\text { Multiphase, } \\
\text { mixed-methods } \\
\text { explanatory }\end{array}$ & $\begin{array}{l}5 \text { principals } \\
\text { and } 5 \\
\text { ex-teachers } \\
\text { (Australia) }\end{array}$ & $\begin{array}{l}\text { - Semistructured, } \\
\text { qualitative, } \\
\text { individual } \\
\text { interviews }\end{array}$ & $\begin{array}{l}\text { - Content } \\
\text { analysis } \\
\text { - Leadership } \\
\text { Matters } \\
\text { framework of } \\
\text { Education } \\
\text { Queensland } \\
\text { (involving five } \\
\text { domains of } \\
\text { leadership: } \\
\text { personal, } \\
\text { relational, } \\
\text { intellectual, } \\
\text { organizational } \\
\text { and } \\
\text { educational } \\
\end{array}$ & $\begin{array}{l}\text { - Relational leadership } \\
\text { practices were found to } \\
\text { have the strongest impact } \\
\text { on teachers' work } \\
\text { commitment. } \\
\text {-Significant differences } \\
\text { were established between } \\
\text { principal and teacher } \\
\text { groups: The second most } \\
\text { important practice was } \\
\text { personal leadership } \\
\text { according to the teachers, } \\
\text { while principals rated } \\
\text { organizational and } \\
\text { educational leadership } \\
\text { practices as the second }\end{array}$ \\
\hline
\end{tabular}




\begin{tabular}{|c|c|c|c|c|c|}
\hline & & & & leadership) & $\begin{array}{lr}\text { most important } & \text { for } \\
\text { teachers' } & \text { work } \\
\text { commitment. } & \end{array}$ \\
\hline \multicolumn{6}{|c|}{$\begin{array}{l}\text { Heidmets, M., \& Liik, K. (2014). School principals' leadership style and teachers' subjective well-being at school. Problems of Education in } \\
\text { the 21st Century, 62(62) 40-50. }\end{array}$} \\
\hline $\begin{array}{l}\text { Examination of how } \\
\text { school leadership style is } \\
\text { related with several } \\
\text { teacher outcomes } \\
\text { (wellbeing at school, } \\
\text { burnout, job insecurity, } \\
\text { emotional and cognitive } \\
\text { identification with school } \\
\text { and turnover intention). }\end{array}$ & Survey & $\begin{array}{l}305 \text { teachers } \\
\text { from } 12 \\
\text { public } \\
\text { schools in } \\
\text { Estonia }\end{array}$ & $\begin{array}{l}\text { - MLQ version 5x } \\
\text { - Copenhagen } \\
\text { Burnout Inventory } \\
\text { (CBI) } \\
\text { - Job Insecurity } \\
\text { Scale } \\
\text { - Emotional } \\
\text { Identification } \\
\text { Scale } \\
\text {-Turnover } \\
\text { Intention Scale }\end{array}$ & $\begin{array}{l}\text { - Descriptive } \\
\text { statistics } \\
\text { - Spearman's } \\
\text { rank-order } \\
\text { correlation } \\
\text { - ANOVA }\end{array}$ & $\begin{array}{l}\text { Transformational } \\
\text { leadership was superior to } \\
\text { transactional leadership, } \\
\text { driving teachers to } \\
\text { identify themselves with } \\
\text { their school emotionally } \\
\text { and cognitively to a } \\
\text { greater extent; feel lower } \\
\text { levels of job insecurity, } \\
\text { burnout and turnover } \\
\text { intentions. }\end{array}$ \\
\hline
\end{tabular}

Josanov-Vrgovic, N., \& Pavlovic, N. (2014). Relationship between the school principal leadership style and teachers' job satisfaction in Serbia. Montenegrin Journal of Economics, 10(1), 43-57.

\begin{tabular}{ll|l|l|l|l|l}
$\begin{array}{l}\text { Examination of the } \\
\text { relationship between } \\
\text { principal leadership style } \\
\text { and teacher job } \\
\text { satisfaction. }\end{array}$ & $\begin{array}{l}22 \text { primary } \\
\text { and } \\
\text { secondary } \\
\text { schools in } \\
\text { Serbia. }\end{array}$ & $\begin{array}{l}\text {-Managerial Grid } \\
\text { Model } \\
\text {-Job Satisfaction } \\
\text { Survey (JSS) }\end{array}$ & $\begin{array}{l}\text {-Descriptive } \\
\text { statistics } \\
\text {-Correlation } \\
\text { analysis } \\
\text {-Regression } \\
\text { analysis }\end{array}$ & $\begin{array}{l}\text {-People-oriented } \\
\text { principals were found to } \\
\text { have a positive impact on } \\
\text { teacher job satisfaction in } \\
\text { school development, } \\
\text { management, and } \\
\text { relationship with } \\
\text { colleagues, while } \\
\text { task-oriented principals } \\
\text { had a negative impact on } \\
\text { teacher satisfaction in } \\
\text { inanagement, } \\
\text { communication, and } \\
\text { school development. }\end{array}$ \\
\end{tabular}

Lourmpas, S., \& Dakopoulou, A. (2014). Educational leaders and teachers' motivation for engagement in innovative programmes. The case of Greece. Procedia - Social and Behavioral Sciences 116, 3359-3364

\begin{tabular}{l|l|l|l|l|l}
$\begin{array}{l}\text { Investigation of the } \\
\text { influence of educational } \\
\text { leaders on teachers' } \\
\text { engagement in } \\
\text { innovative programs and } \\
\text { their impact on teachers' } \\
\text { motivation. }\end{array}$ & $\begin{array}{l}\text {-Quantitative and } \\
\text { qualitative }\end{array}$ & $\begin{array}{l}-88 \\
\text { principals } \\
\text { and teachers } \\
\text { in Greece. }\end{array}$ & $\begin{array}{l}\text {-Questionnaires } \\
\text { and structured } \\
\text { interviews }\end{array}$ & $\begin{array}{l}\text { - Descriptive } \\
\text { statistics }\end{array}$ & $\begin{array}{l}\text {-Educational leaders were } \\
\text { found to have an effect on } \\
\text { most of the teachers. }\end{array}$ \\
\end{tabular}

Barrett, C., \& Breyer, R. (2014). The influence of effective leadership on teaching and learning. Journal of Research Initiatives, 1(2).

\begin{tabular}{|c|c|c|c|c|c|}
\hline $\begin{array}{l}\text { Examination of the } \\
\text { impact of principals' } \\
\text { instructional strategies } \\
\text { on teachers' passion and } \\
\text { motivation of academic } \\
\text { achievement. }\end{array}$ & Experimental (?) & $\begin{array}{l}\text {-One } \\
\text { elementary } \\
\text { school in } \\
\text { Southeastern } \\
\text { America (41 } \\
\text { teachers, } 12 \\
\text { teacher } \\
\text { assistants, } 1 \\
\text { principal, } 1 \\
\text { principal } \\
\text { assistant, } \\
654 \\
\text { students) }\end{array}$ & $\begin{array}{l}\text {-Implementation } \\
\text { of several } \\
\text { strategies } \\
\text { (productive } \\
\text { learning, } \\
\text { professional } \\
\text { development } \\
\text { training, } \\
\text { pedagogic } \\
\text { training) }\end{array}$ & $\begin{array}{l}\text {-Observation } \\
\text { and } \\
\text { documentation } \\
\text { of how } \\
\text { frequent the } \\
\text { new strategies } \\
\text { were } \\
\text { implemented. }\end{array}$ & $\begin{array}{lr}\text {-Effective } & \text { teaching } \\
\text { strategies provided by } \\
\text { principals were found to } \\
\text { improve } \\
\text { professional development, } \\
\text { morale and motivation, } \\
\text { leading to enhanced } \\
\text { student achievement. }\end{array}$ \\
\hline
\end{tabular}

Cohen, E. (2015). Principal leadership styles and teacher and principal attitudes, concerns and competencies regarding inclusion. Procedia Social and Behavioral Sciences, 186, $758-764$

\begin{tabular}{|c|c|c|c|c|c|}
\hline $\begin{array}{l}\text { Examination } \\
\text { principal's of } \\
\text { styles and inclusion } \\
\text { attitudes of both } \\
\text { principals and teachers. } \\
\text { needed for successful } \\
\text { inclusion }\end{array}$ & Survey & $\begin{array}{l}15 \\
\text { principals } \\
\text { and } 81 \\
\text { classroom } \\
\text { teachers } \\
\text { from } \\
\text { elementary } \\
\text { schools }\end{array}$ & $\begin{array}{l}\text {-MLQ } \\
\text { Questionnaire } \\
\text {-Attitudes toward } \\
\text { inclusion } \\
\text { questionnaire } \\
\text {-Competencies } \\
\text { scale }\end{array}$ & $\begin{array}{l}\text {-Basic } \\
\text { statistical } \\
\text { analysis } \\
\text { (mean, } \\
\text { standard } \\
\text { deviation and } \\
\text { prevalence) } \\
\text {-Spearman's }\end{array}$ & $\begin{array}{l}\text {-Seniority (years of } \\
\text { teaching experience) was } \\
\text { significantly related to } \\
\text { leadership styles. } \\
\text { - Seniority (years of } \\
\text { teaching experience) was } \\
\text { negatively related to } \\
\text { positive inclusion }\end{array}$ \\
\hline
\end{tabular}




\begin{tabular}{|c|c|c|c|c|c|}
\hline & & (USA) & & $\begin{array}{l}\text { rank } \\
\text { correlation }\end{array}$ & attitudes. \\
\hline \multicolumn{6}{|c|}{$\begin{array}{l}\text { Secong, S.A., Futalan, E.C., \& Aunzo, R.T. (2015). School administrators' management styles in relation to their teachers' performance. } \\
\text { International Journal for Research in English Language, Literature and Humanities, 2(2), 41-54. }\end{array}$} \\
\hline $\begin{array}{l}\text { Investigation } \\
\text { school whether } \\
\text { principals' } \\
\text { management } \\
\text { relyles are } \\
\text { performance. }\end{array}$ & Descriptive-correlational & $\begin{array}{l}52 \quad \text { public } \\
\text { elementary } \\
\text { school } \\
\text { principals in } \\
\text { Bayawan } \\
\text { City, } \\
\text { Philippines }\end{array}$ & $\begin{array}{l}\text { - Raybould's } \\
\text { Management Style } \\
\text { Questionnaire } \\
\text { (autocratic, } \\
\text { bureaucratic, } \\
\text { democratic, } \\
\text { laissez-faire and } \\
\text { paternalistic } \\
\text { styles) } \\
\text {-Performance } \\
\text { Evaluation Sheet } \\
\text { (PES) }\end{array}$ & $\begin{array}{l}\text {-Descriptive } \\
\text { statistics } \\
\text {-Correlation } \\
\text { analysis }\end{array}$ & $\begin{array}{l}\text { Democratic } \\
\text { was slightly neadership } \\
\text { related with teachers' } \\
\text { performance. } \\
\text { Bureaucratic, paternalistic, } \\
\text { and democratic styles } \\
\text { were highly used by } \\
\text { principals, whereas } \\
\text { laissez-faire and autocratic } \\
\text { styles were moderately } \\
\text { used. } \\
\text { Among five styles, only } \\
\text { democratic style was } \\
\text { negatively related with } \\
\text { teacher performance. }\end{array}$ \\
\hline \multicolumn{6}{|c|}{$\begin{array}{l}\text { Hallinger, P. (2016). Leadership and teacher learning in urban and rural schools in China: Meeting the dual challenges of equity and } \\
\text { effectiveness. International Journal of Educational Development, 51,163-173. }\end{array}$} \\
\hline $\begin{array}{l}\text { Investigation of } \\
\text { differences related to } \\
\text { principal leadership and } \\
\text { teacher learning in rural } \\
\text { and urban schools. }\end{array}$ & Cross-sectional survey & $\begin{array}{l}92 \text { urban } \\
\text { teachers and } \\
423 \text { rural } \\
\text { teachers } \\
\text { from } 31 \\
\text { schools in } 3 \\
\text { provinces of } \\
\text { mainland } \\
\text { China }\end{array}$ & $\begin{array}{l}3 \text { scales } \\
\text { (15 subscales) } \\
- \\
\text { Learning-Centered } \\
\text { leadership } \\
\text { - Teacher Agency } \\
-\quad \quad \text { Teacher } \\
\text { Professional } \\
\text { Learning }\end{array}$ & $\begin{array}{l}\text {-Reliability } \\
\text { and validity } \\
\text { analyses } \\
\text {-Confirmatory } \\
\text { factor analysis } \\
\text { (CFA) } \\
\text {-Comparative } \\
\text { fix index } \\
\text { (CFI), } \\
\text {-Standardized } \\
\text { root mean } \\
\text { square residual } \\
\text { (SRMR), root } \\
\text { mean square } \\
\text { error of } \\
\text { approximation } \\
\text { (RMSEA), and } \\
\text { x2 (for } \\
\text { model fit) } \\
\text {-Bootstrapping } \\
\text { method } \\
\text {-Structural } \\
\text { equation } \\
\text { modeling } \\
\text { (SEM) }\end{array}$ & $\begin{array}{l}\text {-Learning-centered } \\
\text { leadership partially } \\
\text { mediated teacher learning. } \\
\text {-There were no significant } \\
\text { differences between rural } \\
\text { and urban teachers. } \\
\text {-Teacher agency and } \\
\text { teacher trust were found to } \\
\text { be mediators. }\end{array}$ \\
\hline
\end{tabular}

\section{Discussion}

Schools are places for learning, which are not oriented just at students, but also for teachers, parents, staff and even the entire environment of the school. This fact renders schools become highly important organizations particularly in terms of their management. Schools are closely related with sustainable economic and social improvement. Thus, they have to change according to the changing requirements of the society, which also requires transformation and redefinition of school principals.

Principals, as the key component in an educational institution, are supposed to create a clear road map to be followed by all school members. Although effectiveness and success of a school depend on several factors, it is obvious that principals have to undertake the leader role and work together with teachers. From this standpoint of view, the objective of this paper was to examine the recent topics of research and variables associated with principal leadership and teachers' work attitudes in both Turkey and foreign countries over the last six years (2010-2016). It is believed that this paper can contribute to future research in terms of focusing on the gaps in this specific literature by providing a collective summary of recent studies from different countries regarding educational management, especially in elementary schools. 
The review of the selected studies pointed out particular differences and similarities in the leadership research between Turkish and foreign studies. First, the research on principal leadership in elementary schools from Turkey seems to concentrate mostly on instructional leadership rather than other styles that are most commonly discussed in the field of organizational management. The foreign studies, on the other hand, have explored a wider range of leadership styles such as transformational, democratic, autocratic, and authentic leadership instead of focusing on a single type of leadership, which are usually discussed in relation to organizations other than educational institutions. Second, both Turkish and foreign studies seem to investigate a wide spectrum of research variables related to teachers, including job satisfaction, job performance, and commitment; namely work attitudes of teachers. Third, it is seen that Turkish studies have invested more in how the leadership behaviors are perceived by the principals and teachers, which was usually found to differ greatly. For instance, principals are likely to report that they display the behaviors of the leadership style measured, whereas teachers have significantly different opinions (e.g., Ayık \& Şayır, 2014; Al Cihabi \& Al Chiabi, 2014). Foreign studies, in turn, seem to compare certain leadership behaviors in terms of certain teacher outcomes. For example, the study by Heidmets and Liik (2004) from Estonia investigated transformational leadership versus transactional leadership style, while Secong et al. (2014) from Philippines explored five different styles of leadership (autocratic, bureaucratic, democratic, laissez-faire and paternalistic styles).

Despite the differences, Turkish and foreign studies seem to have some similarities, too. One prominent aspect of both Turkish and foreign studies is that principals are likely to be insufficient in providing teachers with necessary tools of support, improvement and professional development, regardless of the leadership style in question (e.g. Korkmaz, 2015; Josanov-Vrgovic, \& Pavlovic, 2014). Yet, teachers' work attitudes such as job performance, job commitment, and work engagement are known to be considerably influenced by the leadership behaviors of administrators. For instance, the study by Ereş and Akyürek (2016) established that distributed leadership was positively related with teachers' job satisfaction, while Adeyemi (2010) concluded that teachers display better performance with autocratic principals compared to democratic or laissez-faire leaders. Despite the differences in leadership styles, it is obvious that teachers expect support and recognition from their administrators, which would reflect on their teaching performance and ultimately the overall success of schools. Another similarity is that almost all selected studies employed similar methods of data collection and analysis. Only one study from America (Barrett, \& Breyer, 2014) used different methodology that involved implementation and observation of various strategies on instructional leadership. Besides that, all studies were conducted using survey method, which can limit the generalization of research findings and involve self-report bias.

\section{Conclusion}

It is clear that all countries seek to develop a strategy for effective schools and doing research on how to achieve this. There is a vast body of research proving the significant influence of principal leadership on especially teacher satisfaction (e.g. Tillman \& Tillman, 2008; Sharma \& Jyoti, 2006). It is understandable why teacher satisfaction has been discussed so much since unsatisfied teachers are likely to exhibit poor performance, which eventually leads to undesirable effects on student and school achievement. Other than satisfaction, all teacher outcomes may be associated with principals due to the principals' key role in an educational institution. Therefore, the relationship between principal leadership and its influence on teachers is still a topic to be studied in several perspectives. This paper, however, focused on a structured framework in exploring such relationship. In this sense, it should be noted that this paper tried to select studies from countries with different characteristics in order to capture a broad perspective; however, most of the foreign studies are from the Western countries that are usually known to have a good education system. Nevertheless, educational systems may differ among the countries and this may affect the leadership style to be discussed.

This paper concluded that there is still room to discover in principal leadership around the world. No research seems to offer a certain definition of effective leadership in schools. The review of the studies within the scope of this research demonstrates different perceptions in leadership behaviors between principals and teachers. This may indicate a valuable outcome because either principals or the teachers may not be fully aware of the behaviors displayed by a certain leadership style. Therefore, such difference can be examined in future studies in order to establish which factors lead to this difference in perceptive. Additionally, both school principals and teachers can be trained on the leadership style believed to be exhibited by the principal, and then, the perceptions of both parties can be measured in order to determine whether such difference still exists. In this regard, more studies that are experimental will be helpful in discovering which leadership practices produce greater effectiveness. Furthermore, even though the practice is challenging, longitudinal studies can be developed for examining a particular leadership style in detail. Such research, if possible, would offer very valuable and 
in-depth information, especially on the process from the onset of principal leadership behaviors toward the outcomes at the teacher level.

Moreover, as the studies were selected from different countries, it is possible to consider them all country- and culture-specific. Thus, cross-cultural studies can be conducted to explore whether the supportive and improving environment results from the characteristics of the culture, education system or the style of leadership adopted by principals. Findings of such studies may involve comparison with countries considered to have a high-quality education system. This kind of studies may even consider the focus findings of international student assessment systems such as PISA in order to relate the results with student outcomes.

In light of the review of the selected studies, this paper concludes that every school should formulate its own management strategy considering their specific characteristics. In such process, principals should consider the expectations of teachers and its implications on overall school success. Due to its great importance, particularly elementary school principals should develop themselves by learning various leadership styles and their implementation. Besides, leadership and management programs or seminars provided during in-service training would be beneficial in terms of both principals and teachers.

\section{References}

Adeyemi, T. O. (2010). Principals' leadership styles and teachers' job performance in senior secondary schools in Ondo State, Nigeria. Current Research Journal of Economic Theory, 3(3), 84-92.

Aghenta, J. A. (2001). Educational planning: A turning point in education and development in Nigeria. Inaugural Lecture Series 58 University of Benin, Benin-City, Nigeria, 10-18.

Aybek, B., Titiz, H., \& Gümüşay, T. (2014). İlkokul müdürlerinin etkili liderlik düzeylerine ilişkin öğretmen görüşlerinin incelenmesi. Journal of Research in Education and Teaching, 3(4), 342-355.

Ayık, A., \& Şayir, G. (2014). İlköğretim kurumlarında görevli okul müdürlerinin öğretimsel liderlik davranışlarının çeşitli değişkenler açısından incelenmesi. Ekev Akademi Dergisi, 18(60), 15-30.

Balkar, B. (2015). Okul müdürlerinin kültürel liderlik davranışlarının simetrik ve asimetrik okul kültürü bağlamında incelenmesi. International Journal of Science Culture and Sport (IntJSCS), 4, 274-287. https://doi.org/10.14486/IJSCS389

Barrett, C., \& Breyer, R. (2014). The influence of effective leadership on teaching and learning. Journal of Research Initiatives, $1(2)$.

Başaran, İ. E. (2000). Yönetim. Anara: Umut Yayım Dağıtım.

Bellibas, M. S., Bulut, O., Hallinger, P, \& Wang, W. C. (2016). Developing a validated instructional leadership profile of Turkish primary school principals. International Journal of Educational Research, 75, 115-133. https://doi.org/10.1016/j.jijer.2015.10.002

Çelik, V. (1999). Eğitimsel Liderlik. Ankara: Pegem Yayınc1lık.

Cerit, Y. (2012a). Paternalistik liderlik ile yöneticiden ve işin doğasından doyum arasındaki ilişki. Ondokuz Mayıs Üniversitesi Eğitim Fakültesi Dergisi, 31(2), 35-56. https://doi.org/10.7822/egt11

Cerit, Y. (2012b). Lider Üye Etkileşimi İle Öğretmenlerin Performansları Arasındaki İlişki. Balikesir University The Journal of Social Sciences Institute, 15(28), 33-45.

Cohen, E. (2015). Principal Leadership Styles and Teacher and Principal Attitudes, Concerns and Competencies regarding Inclusion. Procedia - Social and Behavioral Sciences, 186, 758-764. https://doi.org/10.1016/j.sbspro.2015.04.105

Danielson, C. (2010). Evaluations that help teachers learn. Educational Leadership, 68(4), 35-39.

Ereş, F., \& Akyürek, M. İ. (2016). Relationship levels between distributed leadership behaviors of principals and the teachers' perception of job satisfaction. GEFAD / GUJGEF, 36(3), 427-449.

Farr, S. (2011). Leadership, not magic. Educational Leadership, 68(4), 28-33.

Finnigan, K. S. (2010). Principal Leadership and teacher motivation under high-stakes accountability policies. Leadership and Policy in Schools, 9(2), 161-189. https://doi.org/10.1080/15700760903216174

Graham, K., Hudson, P., \& Willis, J. (2014, December). How can principals enhance teacher job satisfaction and work commitment? Paper presented at the Australian Association of Research in Education (AARE) Conference, Brisbane, Australia. 
Hallinger, P. (2016). Leadership and teacher learning in urban and rural schools in China: Meeting the dual challenges of equity and effectiveness. International Journal of Educational Development, 51, 163-173. https://doi.org/10.1016/j.ijedudev.2016.10.001

Heidmets, M., \& Liik, K. (2014). School principals' leadership style and teachers' subjective well-being at school. Problems of Education in the 21st Century, 62(62) 40-50.

İlgar, L. (2000). Eğitim yönetimi okul yönetimi sinıf yönetimi. İstanbul: Beta Yayınc1lık.

İnand1, Y., Ağgün, N., \& Atik, Ü. (2010). Yönetici ve öğretmenlerin görüşlerine göre ilköğretim okullarında çalışan öğretmenlerin iş doyum düzeyleri. Mersin University Journal of the Faculty of Education, 6(1), 102-126. Retrieved from https://10.17860/efd.46861

Jasbi, A. (2011). Management principles and basics. Second Printing, Hakimbashi publication. Tehran.

Josanov-Vrgovic, N., \& Pavlovic, N. (2014). Relationship between the school principal leadership style and teachers' job satisfaction in Serbia. Montenegrin Journal of Economics, 10(1), 43-57.

Kabaklı Çimen, L. (2015). The comparison of family functions and occupational performance levels of teachers. The Journal of International Social Researh, 8(37), 759-772. https://doi.org/10.17719/jisr.20153710642

Kahraman, Ü., \& Çankaya, İ. (2015). İlkokullarda performans yönetimi uygulamaları ve öğretmenlerin örgütsel adalet algıları arasındaki ilişki. Turkish Studies International Periodical For The Languages, Literature and History of Turkish or Turkic, 10(7), 519-540. https://doi.org/10.7827/TurkishStudies.8138

Khalkhali, A., Khalatbary, J., \& Azany, M. (2011). The relationship between educational philosophy and leadership style of school principals. Journal of Educational Administration, a new approach. Second $\operatorname{Year}(2), 40-33$.

Korkmaz, İ. (2015). Öğretmenlerin mesleki gelişimlerinde okul yöneticileri ve denetmenlerin etkililiğinin incelenmesi. Uluslararası Ĕgitim Bilimleri Dergisi, 2(4), 55-64. https://doi.org/10.16991/INESJOURNAL.84

Leithwood, K., \& Jantzi, D. (2006). A critical review of the parent engagement literature. Toronto, ON: Ministry of Education.

Leithwood, K., \& Steinbach, R. (1993). The consequences for school improvement of differences in principals' problem-solving processes. In C. Dimmock (Ed.), School-based management and school improvement. New York, NY: Routledge. Retrieved from https://10.0.16.228/9781315824727-11

Lourmpas, S., \& Dakopoulou, A. (2014). Educational leaders and teachers' motivation for engagement in innovative programmes. The case of Greece. Procedia - Social and Behavioral Sciences, 116, 3359-3364. https://doi.org/10.1016/j.sbspro.2014.01.764

Madenoğlu, C., Uysal, Ş., Sarıer, Y., \& Banoğlu, K. (2014). Okul müdürlerinin etik liderlik davranışları ile öğretmenlerin iş doyumlarının örgütsel bağlllıkla ilişkisi. Educational Administration: Theory and Practice, 20(1), 47-69. https://doi.org/10.14527/kuey.2014.003

Ming-Ten, T., Chung-Lin, T., \& Yi-Chou, W. (2011). A study on the relationship between leadership style, emotional intelligence, self-efficacy and organizational commitment A case study of the Banking Industry Taiwan. African Journal of Business Management, 5(13), 5319-5329. Retrieved from https://10.5897/AJBM10.932

Nworgu, B. G. (1991). Educational Research: Basic Issues and Methodology. Ibadan: Wisdom Publisher Ltd, pp. 51-60.

Obilade, S. O. (1999). Leadership qualities and styles as they relate to instructional productivity. The Manager Department of Educational, University of Ibadan, 5(1), 25-32.

Okeniyi, C.M. (1995). Relationship between leadership problems and school performance in Oyo State secondary schools (Unpublished Master's Thesis). University of Ibadan. Üniversitesi Sosyal Bilimler Dergisi, 3, 266-282.

Peretemode, V. F. (1991). Education administrations: Applied concepts and theoretical perspective. Lagos: Nigeria. Joja Educational Research and Publishers.

Pirkhaefi, A. R. (2009). Styles of leadership, principles, and practices of improving the efficiency of the employees. Tehran: Hezareh Ghoghnoos.

Sağır, M., \& Memişoğlu, S. P. (2012). The perceptıons of admınıstrators and teachers on elemantary school 
admınıstrators' educational leadership roles. Journal of Research in Education and Teaching, 1(2), 1-12.

Secong, S. A., Futalan, E. C., \& Aunzo, R. T. (2015). School administrators' management styles in relation to their teachers' performance. International Journal for Research in English Language, Literature and Humanities, 2(2), 41-54.

Sharma, R. D., \& Jyoti, J. (2006). Job satisfaction among school teachers. IIMB Management Review, 18(4), 349-363.

Şişman, M. (2002). Öğretim liderliği. Ankara: Pegem. Retrieved from https://10.14527/9789756802700

Şişman, M. (2012). Ĕ̈itimde mükemmellik arayışı: etkili okullar. Ankara: Pegem. Retrieved from https://10.14527/9789756802946

Teyfur, M., Beytekin, O. F., \& Yalçınkaya, M. (2013). A research on the ethical leadership of primary school administrators and the organizational trust levels in primary schools: The sample of İzmir. Dicle Üniversitesi Ziya Gökalp Eğitim Fakültesi Dergisi, 21, 84-106.

Tillman, W. R., \& Tillman, C. J. (2008). And you thought it was the apple: A study of job satisfaction among teachers. Academy of Educational Leadership Journal, 12(3), 1-18.

Töremen, F., \& Yasan, T. (2010). İlköğretim okulu yöneticilerinin dönüşümcü liderlik özellikleri (Malatya ili örneği). Pamukkale Üniversitesi Ĕ̆itim Fakültes Dergisi, 20, 27-39.

Townsend, T. (2010). Educating school leaders to think and act both locally and globally. International Journal of Leadership in Education, 13(3), 335-348. https://doi.org/10.1080/13603124.2010.505300

Yavaş Taşdelen, T., Aküzüm, C., Tan, C., \& Uçar, M. B. (2015). Instructional leadership behaviours of school principals in terms of teachers' opinions. Turkish Studies International Periodical For The Languages, Literature and History of Turkish or Turkic, 10(3), 1-26. Retrieved from https://10.7827/TurkishStudies.7843

Yeşilyurt, R. (2015). Okul müdürlerinin etkileşimci liderlik stilleri ile öğretmenlerin okula bağlllık düzeyleri arasındaki ilişki. Yüksek Lisans Tezi. Eskişehir Osmangazi Üniversitesi, Eğitim Bilimleri Enstitüsü, Eskişehir.

Yukl, G. A. (2002). Leadership in organizations (5th ed.). Upper Saddle River, NJ: Prentice-Hall.

\section{Copyrights}

Copyright for this article is retained by the author(s), with first publication rights granted to the journal.

This is an open-access article distributed under the terms and conditions of the Creative Commons Attribution license (http://creativecommons.org/licenses/by/4.0/). 